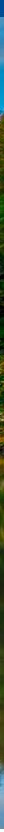

\title{
Migrating Office 365 From ADFS to Ping Federate
}

NLIT 2019

Kevin Conway

May 31, 2019

This manuscript has been authored by Fermi Research Alliance, LLC under Contract No. DE-AC02-07CH11359 with the U.S. Department of Energy, Office of Science, Office of High Energy Physics. 


\section{Agenda}

- Why migrate?

- Pre-Requisites for Migration

- Create the 0365 Connection

- Federated Trust Maintenance

- Testing

- Lessons Learned

- Questions 


\section{Why migrate?}

\section{ADFS Deployment}

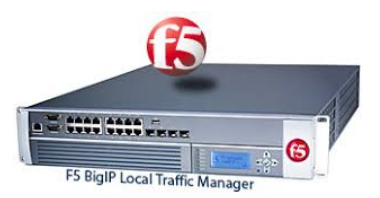

Load Balancer
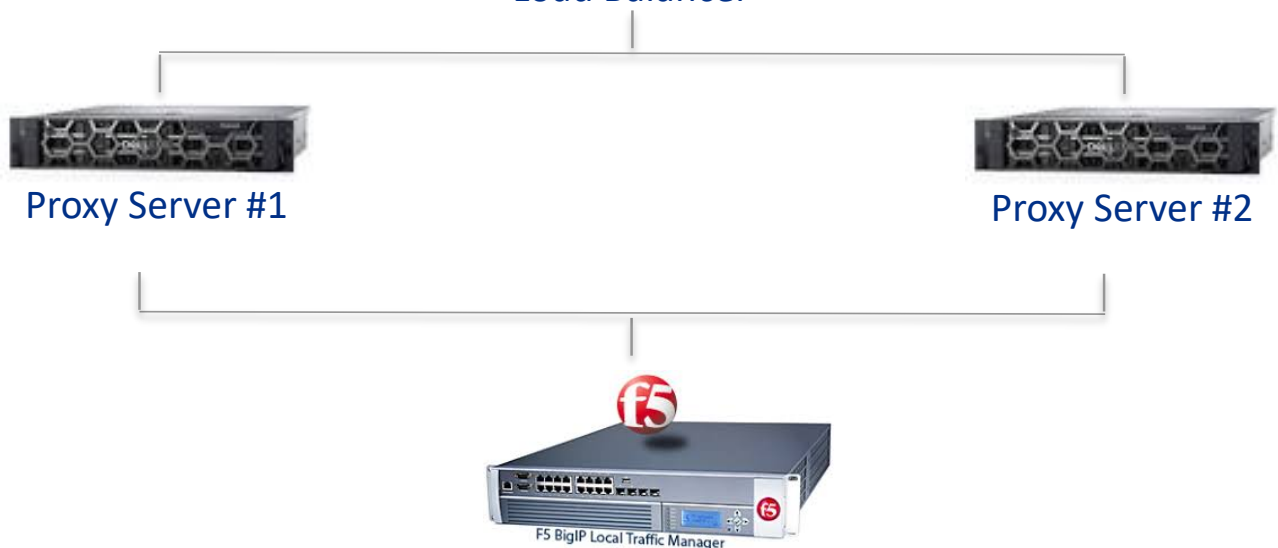

Load Balancer
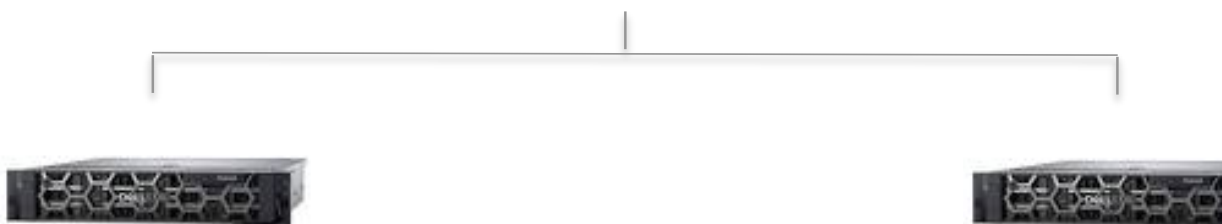

ADFS Server \#1

ADFS Server \#2

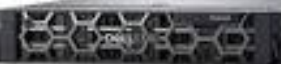

ADFS SQL Server 


\section{Why migrate?}

\section{Ping Federate Deployment}

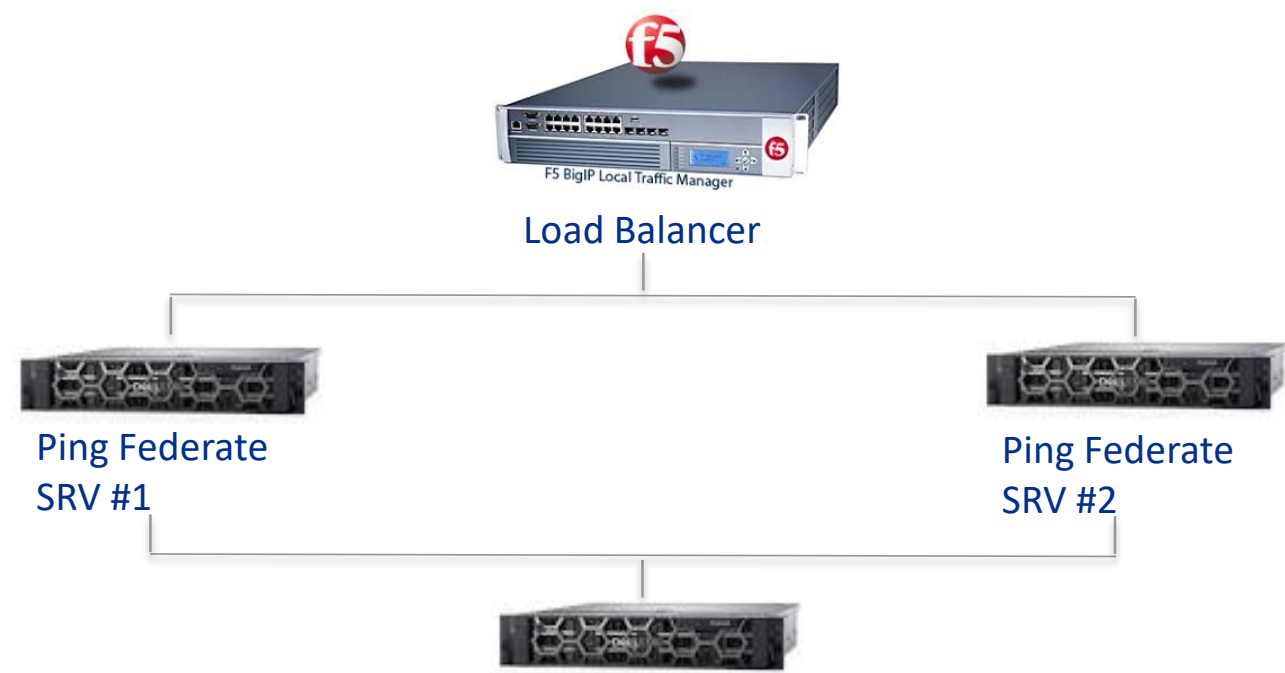

Ping Federate Management Server

- Simple

- Easier to scale

- Cost-effective 
Why migrate?

PingFederate

\section{Added Service Providers!}

Recently added...

s sharePoint

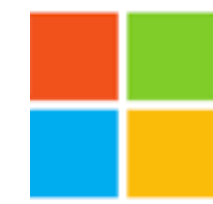

Microsoft

Active Directory

Federation Services

The last remaining SP...

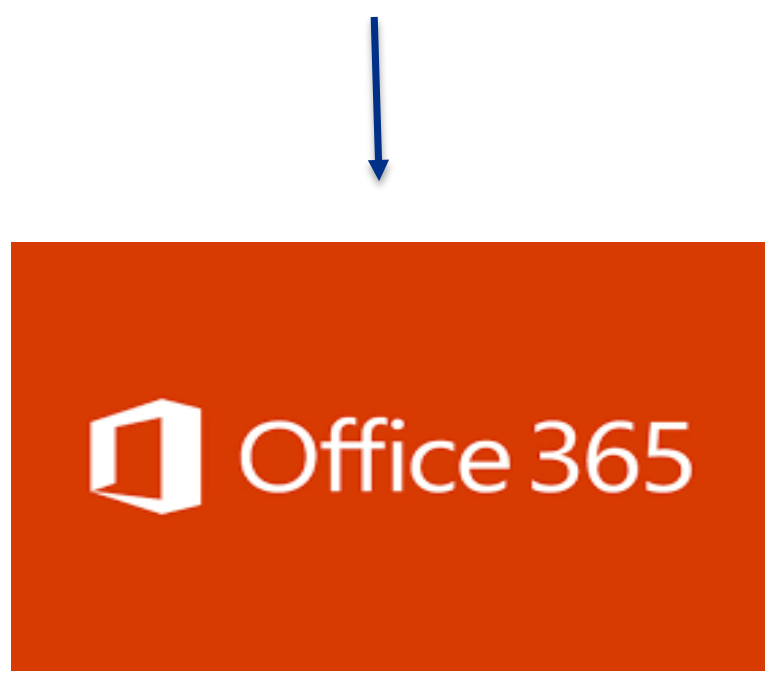




\section{Pre-Requisites for Migration}

\section{Office 365}

Office 365 Tenant (Test Tenant makes life easier!)

- Global Admin Account

\section{PingFederate}

Ping Federate version 8.4 (Recommend version 9.X)

- Admin Account - Full Rights to Management Console

Azure Ad Connect version 1.1.880.0 08

PingFederate Integration with Azure Active Directory and Office 365

Updated June 18, 2018

https://docs.pingidentity.com 


\section{Create the 0365 Connection}

\section{High Level Steps}

- Preparing your Ping Federate Environment

- Create an O365 Connection in Ping Federate Development

- Copy the Connection Settings into Ping Federate Production - API Interface

- Break the ADFS Trust -PowerShell

- Federate Domain with Ping Federate - Use Azure Ad Connect

- Test your O365 Connection - Browsers, Mobile, \& Client Applications

\section{Ping PingFederate}

\section{Existing Settings Used}

Adapter

Data Stores

Signing Certificate
Items needed to Add/Configure

WS-Trust Protocol

Token Processor

Create Credential Validator for upn

Enable objectGUID as binary attribute in datastore 


\section{LDAP Identity Attribute Mapping}

Attribute Contract Fulfillment

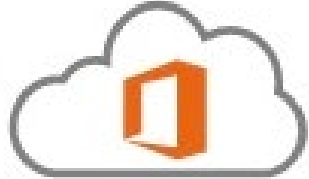

Office 365 Im mutable ID

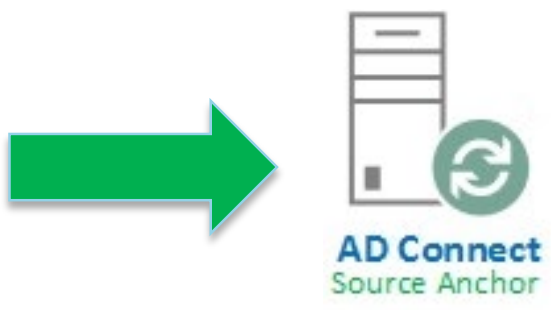

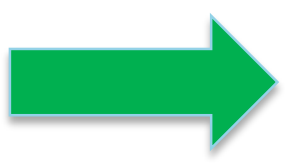

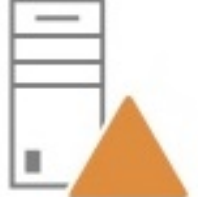

Active Directory

ObjectGUID

Fulfill your Attribute Contract with values from the incoming token, data stores, or dynamic text values.

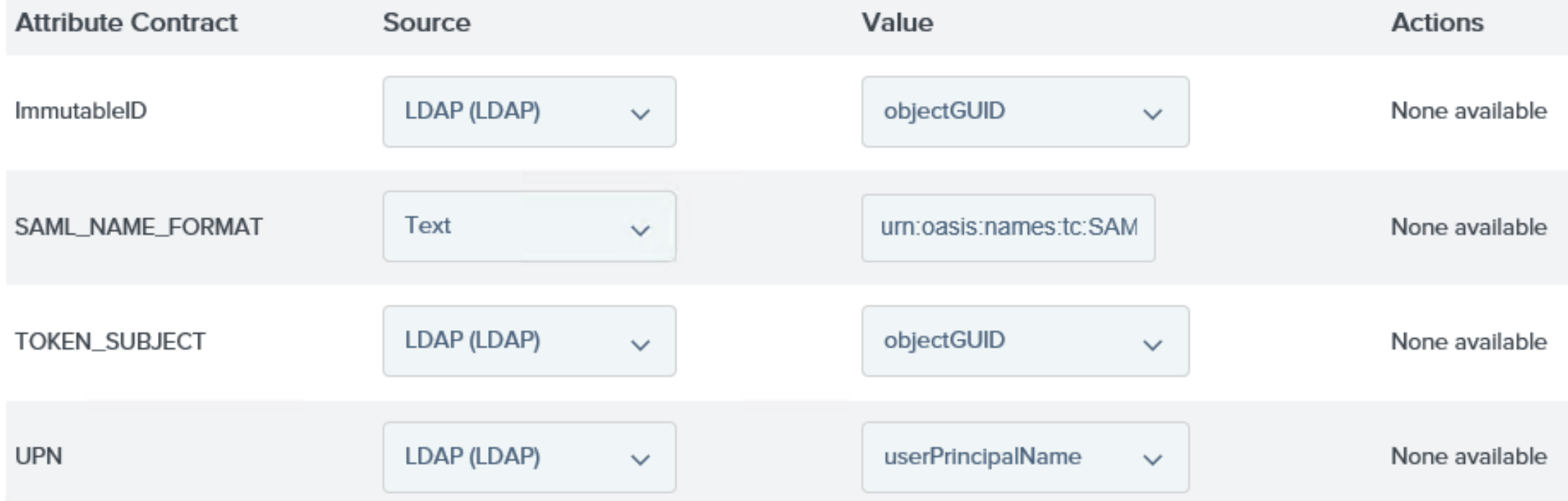




\section{Enable the WS-Trust Protocol}

Enable the WS-Trust Protocol in Server Settings on The Ping Management Server Interface Server Settings

\begin{tabular}{|c|c|c|c|c|c|c|}
\hline System Info & \multicolumn{2}{|c|}{ Runtime Notifications } & \multicolumn{2}{|c|}{ Runtime Reporting } & \multicolumn{2}{|c|}{ Roles \& Protocols } \\
\hline WS-Trust S & ting & Outbound & isioning & Metada & Signing & Metadata \\
\hline
\end{tabular}

\section{Enable for Identity Providers}

ENABLE IDENTITY PROVIDER (IDP) ROLE AND SUPPORT THE FOLLOWING:

$\checkmark$ SAML 2.0

AUTO-CONNECT PROFILE

SAML 1.1

SAML 1.0

$\checkmark$ WS-FEDERATION

$\checkmark$ OUTBOUND PROVISIONING

$\checkmark$ WS-TRUST 


\section{Enable the WS-Trust Protocol}

Enable WS-Trust Protocol in Server Settings $\rightarrow$ Connection Type for the Office 365 Connection

WS Fed Office 365 Test1

\section{SP Connection}
Connection Type
Connection Options
General Info
Browser SSO
WS-Trust STS

Select the type of connection needed for this SP: Browser SSO Profiles (for Browser SSO), WS-Trust STS (for Outbound Provisioning (for provisioning users/groups to an SP) or all.

\section{CONNECTION TEMPLATE \\ No Template \\ $\checkmark$ BROWSER SSO PROFILES \\ PROTOCOL \\ WS-FEDERATION \\ SAML 1.1}

$\checkmark$ WS-TRUST STS

OUTBOUND PROVISIONING

Note! You may receive an error when running through The Azure AD Connect Wizard that it requires WS-TRUST Protocol and will not proceed until its selected In the Management Console. Ping Documentation seemed incorrect here. WS-Trust Protocol was required to complete the Federated Trust with Ping Federate. 


\section{Create Token Processor instance for WS-TRUST}

\section{From the Identity Provider Page select Token Processors}

\section{Manage Token Processor Instances}

Token processors validate incoming tokens when PingFederate is acting as a security token service (STS). Create instances of token processors here that can then be used in STS SP connections or token translator mappings.

$\begin{array}{llll}\text { Instance Name } \hat{\imath} & \text { Instance ID } & \text { Type } & \text { Parent Name } \\ \text { SAML2 } & \text { SAML2 } & \text { SAML 2.0 Token Processor } & \text { None Available - In Use } \\ \text { WS-TRUST } & \text { WSTRUST } & \text { Username Token Processor } & \text { None Available - In Use }\end{array}$

\section{Create Token Processor Instance}

Type

Instance Name

Instance ID

Type

Class Name

Parent Instance Name

Instance Configuration

Credential Validators

Credential Validators

Authentication Attempts

Extended Contract

Attribute

\section{Summary}

WS-TRUST

WSTRUST

Username Token Processor

com.pingidentity.pf.tokenprocessors.username.UsernameTokenProcessor

None

FERMITEST

Credential Validators

FERMITEST Samaccountname Are configured here
Type -

Username

username 


\section{Create the Credential Validator for UPN}

Configure a Password Credential Validator that uses UPN

\section{Manage Token Processor Instances | Create Token Processor Instance}

\begin{tabular}{|l|l|l|l|l}
\hline Type & Instance Configuration & Extended Contract & Token Attributes & Summary \\
\hline
\end{tabular}

- Used to verify username/password pairs in various contexts

- We had one instance created for sAMAccountName=\$\{username $\}$

- We needed to add an instance for UserPrincipalName $=\$\{$ username $\}$

Manage Password Credential Validators

Field Name

LDAP DATASTORE

SEARCH BASE

SEARCH FILTER
Field Value
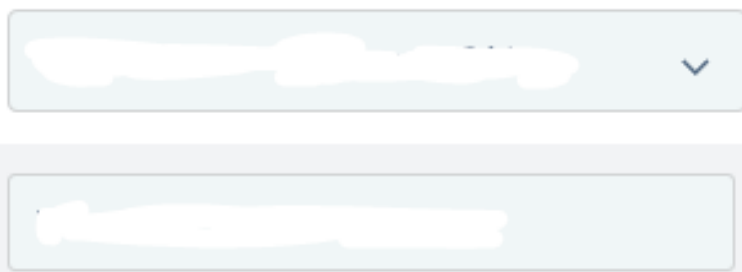

userprincipalname $=\$\{$ username $\}$

\section{Description}

Select the LDAP Datastore.

The location in the directory from which the LDAP search begins.

You may use \$[username] as part of the query. Example (for Active Directory): sAMAccountName $=\$$ [username]. 


\section{Enable objectGUID as binary attribute}

From Server Configuration navigate to your Data Store Configurations, choose your Data Store and choose the Advanced LDAP Options $\rightarrow$ LDAP Binary Types

\section{Manage Data Stores | Data Store | Advanced LDAP Options}

Advanced LDAP Options

LDAP Binary Attributes

Specify the LDAP attributes to be handled as binary data.

Binary Attribute Name

objectGUID

tokenGroups

Edit I Delete

Add

Add objectGUID in the Binary Attribute Name filed and select update 


\section{Create the 0365 Connection using API Interface}

Select /idp/spConnections $\rightarrow$ Get

/idp/spConnections

GET /idp/spConnections

Post /idp/spConnections

GET /idp/spConnections/\{id\}

PUT /idp/spConnections/\{id\}

DELeTE /idp/spConnections/\{id\}
List/Expand Operations | Metadata

Get list of SP connections.

Create a new SP connection.

Find SP connection by ID.

Update an SP connection.

Delete an SP connection.

Search by entityid found in Ping Management Interface

\section{/idp/spConnections}

GET

/idp/spConnections

List/Expand Operations

Get list of SP co

\section{Implementation Notes}

Get a list of all the WS-Fed, WS-Trust, SAML1.0, SAML1.1 and SAML 2.0 SP connections.

\section{Parameters}

Parameter

entityId urn.federation:MicrosoftOnline

\begin{tabular}{ll|}
\hline page & integer \\
\hline numberPerPage & integer
\end{tabular}

Description
Entity ID of the connection to query
fetch. (case-sensitive)

Page number to retrieve.

Number of connections per page.
Selecting Try it Out will return only that connection and not all sp connections in the Management Console

Try it out! 


\section{Create the 0365 Connection using the API Interface}

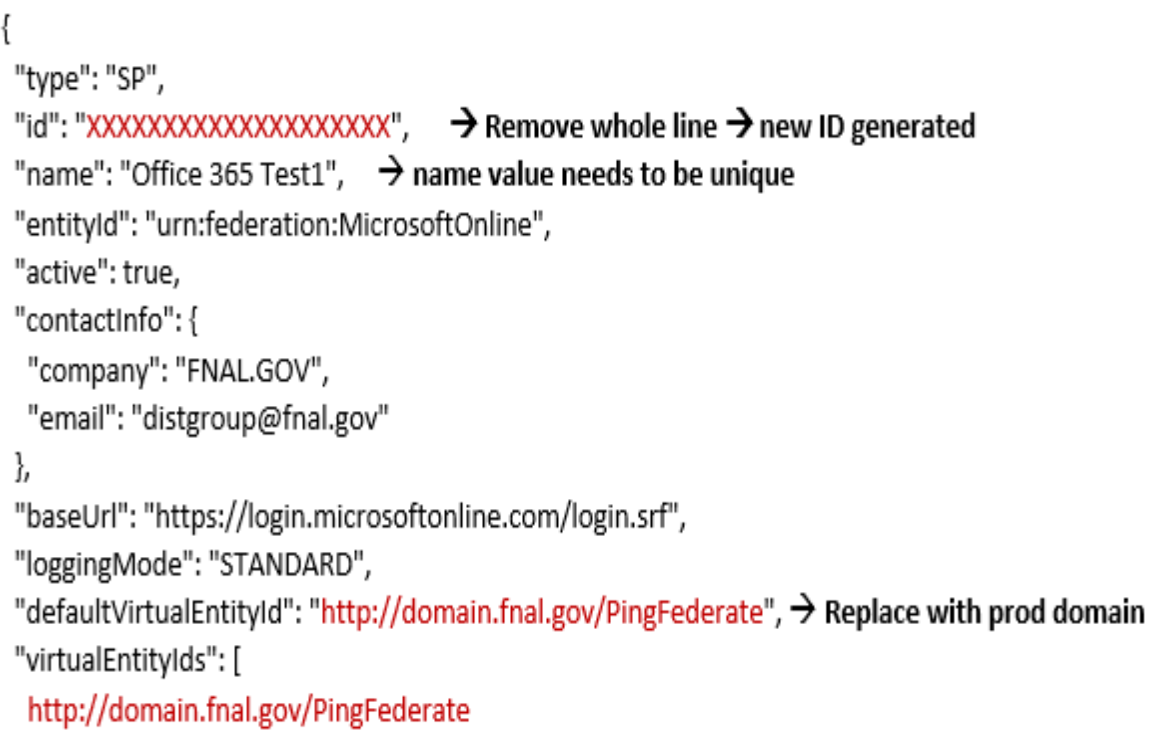

\section{Connection ID and Name ID Values}

In Text Editor you can Edit/Replace values

- "id" value gets generated when connection is created

- "name" value must be unique among SP's

- "virtualEntityID" values refers to Federated Domain

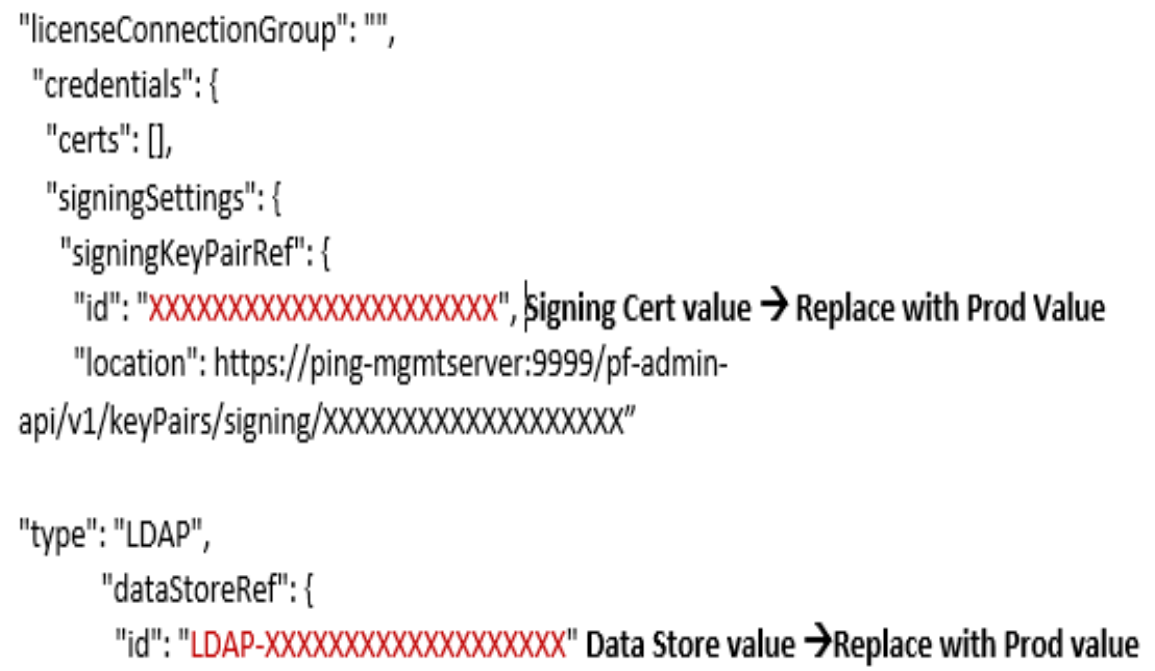

- "id" refers to Signing Certificate value

- "location" refers to Ping Management Server

- "id" LDAP - xxxxxxx refers to Data Store 


\section{Create the 0365 Connection using the API Interface}

Back to the API Interface to paste updated values into the body of new connection field Select $\rightarrow$ POST

\section{Implementation Notes}

Create a new SP connection.

Once Connection is Created, you will find A value for SP "id"

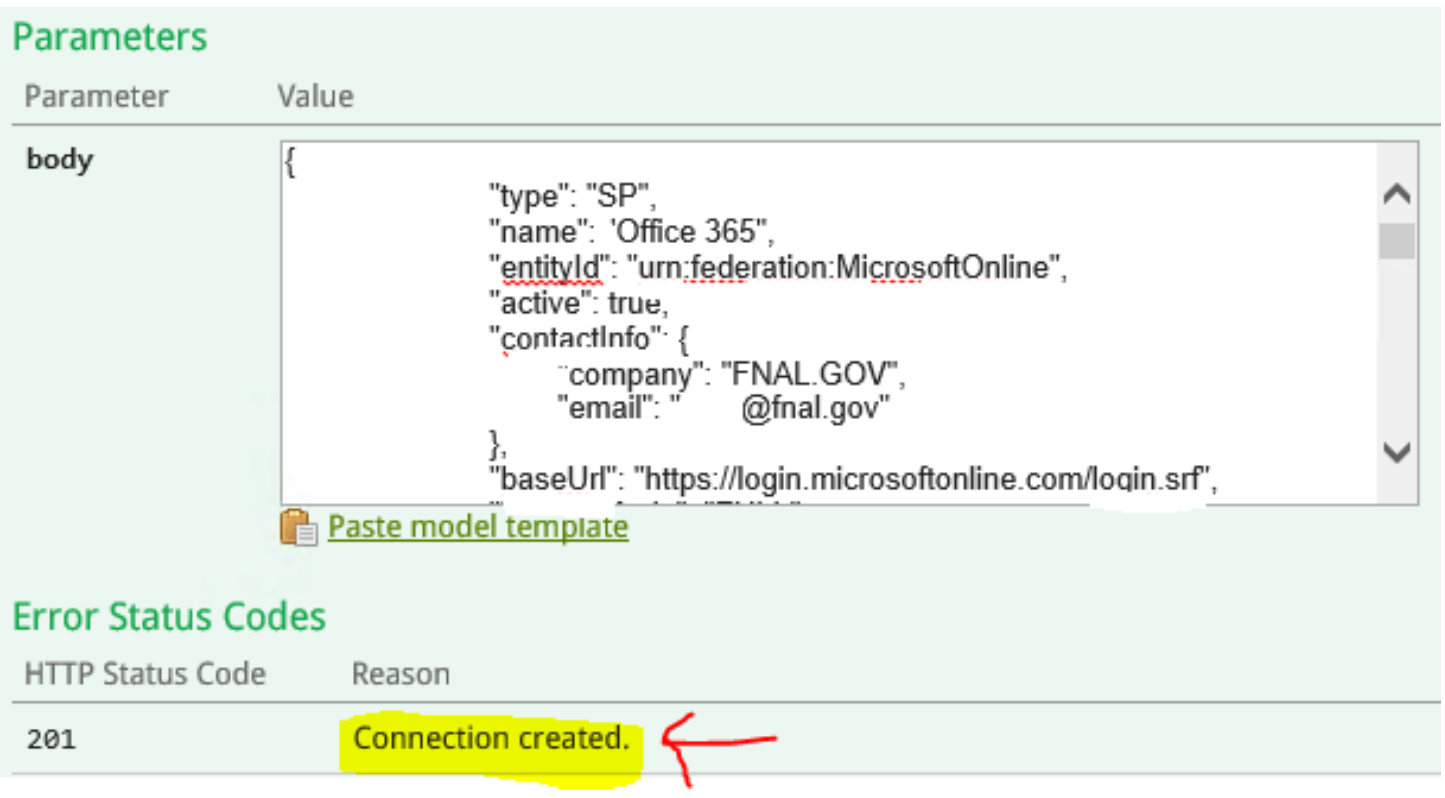

\section{Response Body}

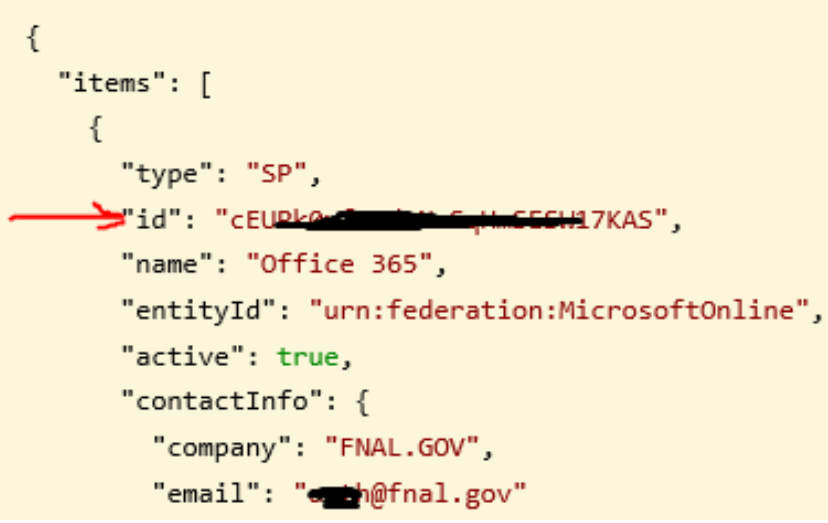

The Connection should now appear in the Ping Management Interface 
\$msolcred $=$ Get-Credential

\#provide credentials cloud service account@domain.onmicrosoft.com

Connect-msolservice -credential \$msolcred

\#At this point, you are authenticated in the cloud tenant

\#Check the current state of the target domain "domain.fnal.gov"

Get-MsolDomain

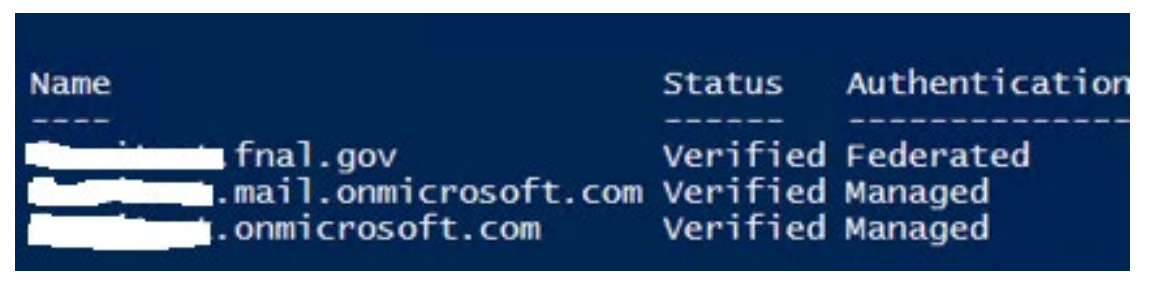

\#Check Federated Domain settings to determine identity Provider

Get-MsolDomainFederationSettings -DomainName 'domain.fnal.gov'

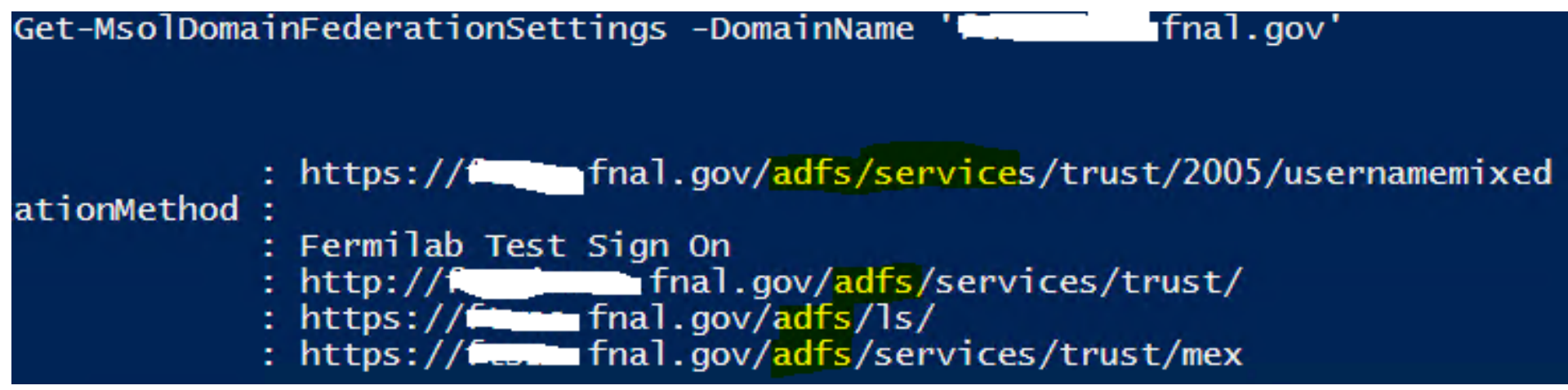




\section{Break the Federated Trust with ADFS}

\#Break the Federated Trust with current identity provider (ADFS)

Set-MsolDomainAuthentication -DomainName domain.fnal.gov -Authentication Managed If successful, No output just prompt below. Trust Broken!!

PS C:IUserslkconway-admin> Get-MsolDomain

Verify Settings after change

\#verify Federated Domain Status is now "managed" and NOT federated

Get-MsolDomain

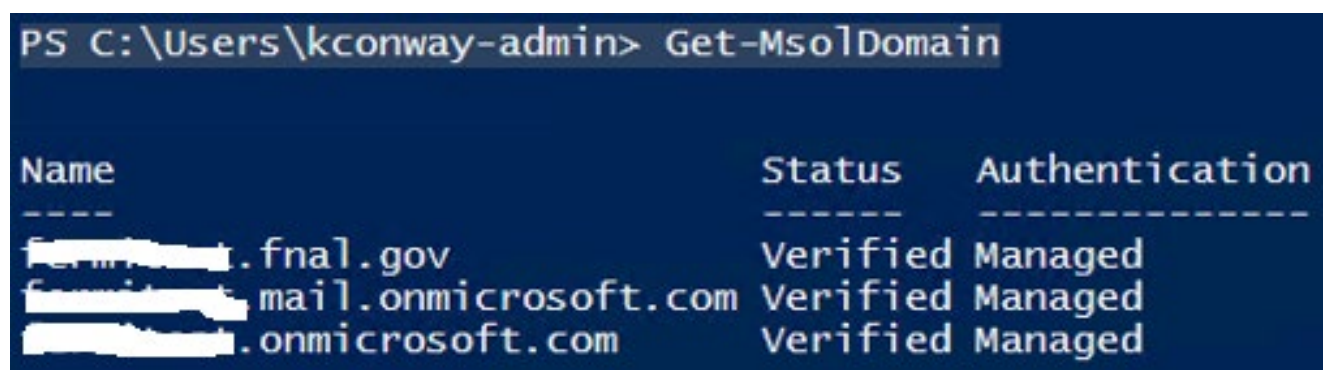

\#check status that there is no listed provider

Get-MsolDomainFederationSettings -DomainName 'domain.fnal.gov' \#No output means no listed provider - This is expected

Proceed to LDAP Server and run Azure Ad Connect to federate with Ping Federate 


\section{Federate Domain with Ping Federate}

Log into LDAP Management Server containing Azure AD Connect Software and run Azure $A D$ Connect.exe

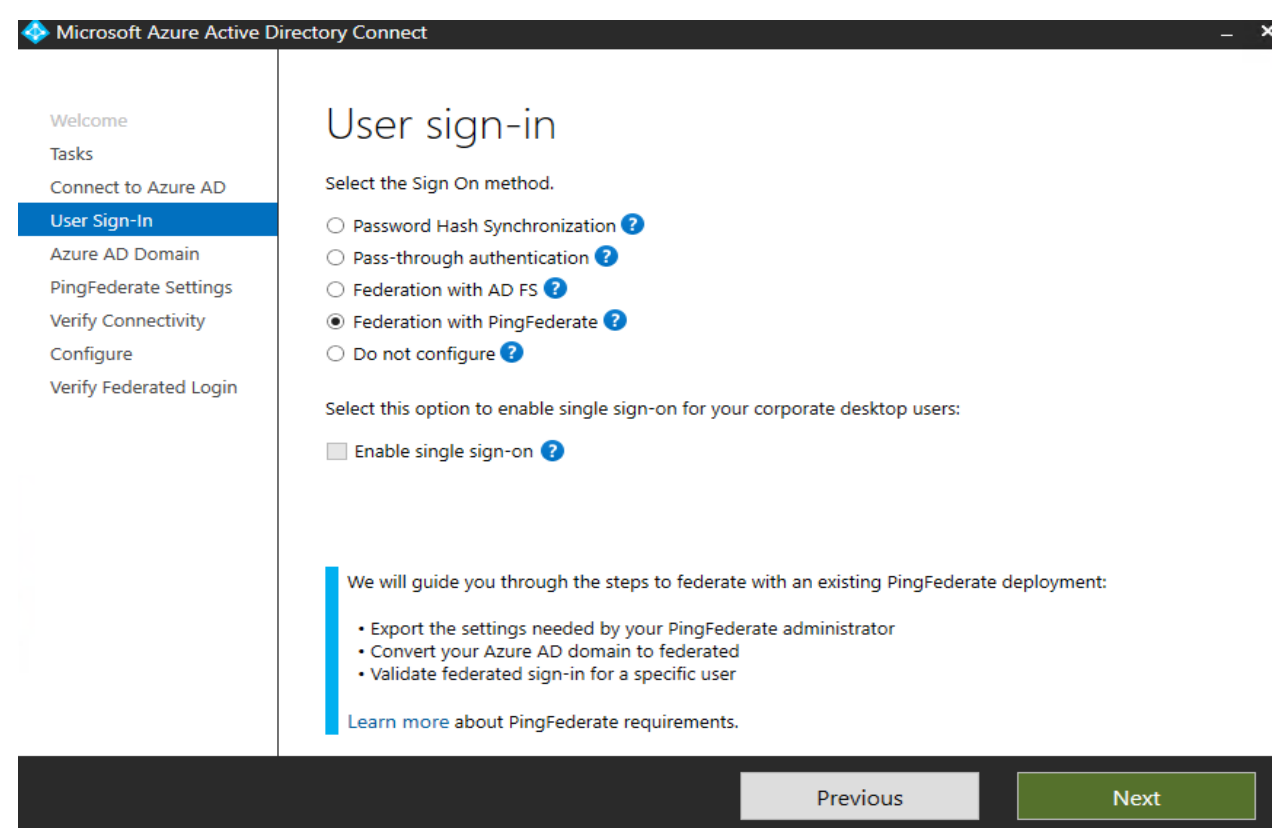

\section{Next $\rightarrow$}

Select your Target domain (domain.gov)

displays message indicating domain is managed and will be converted to a federated domain 


\title{
Federate the Domain with Ping Federate \& export Settings for Ping Management Console
}

\author{
Ping Federate Settings Screen
}

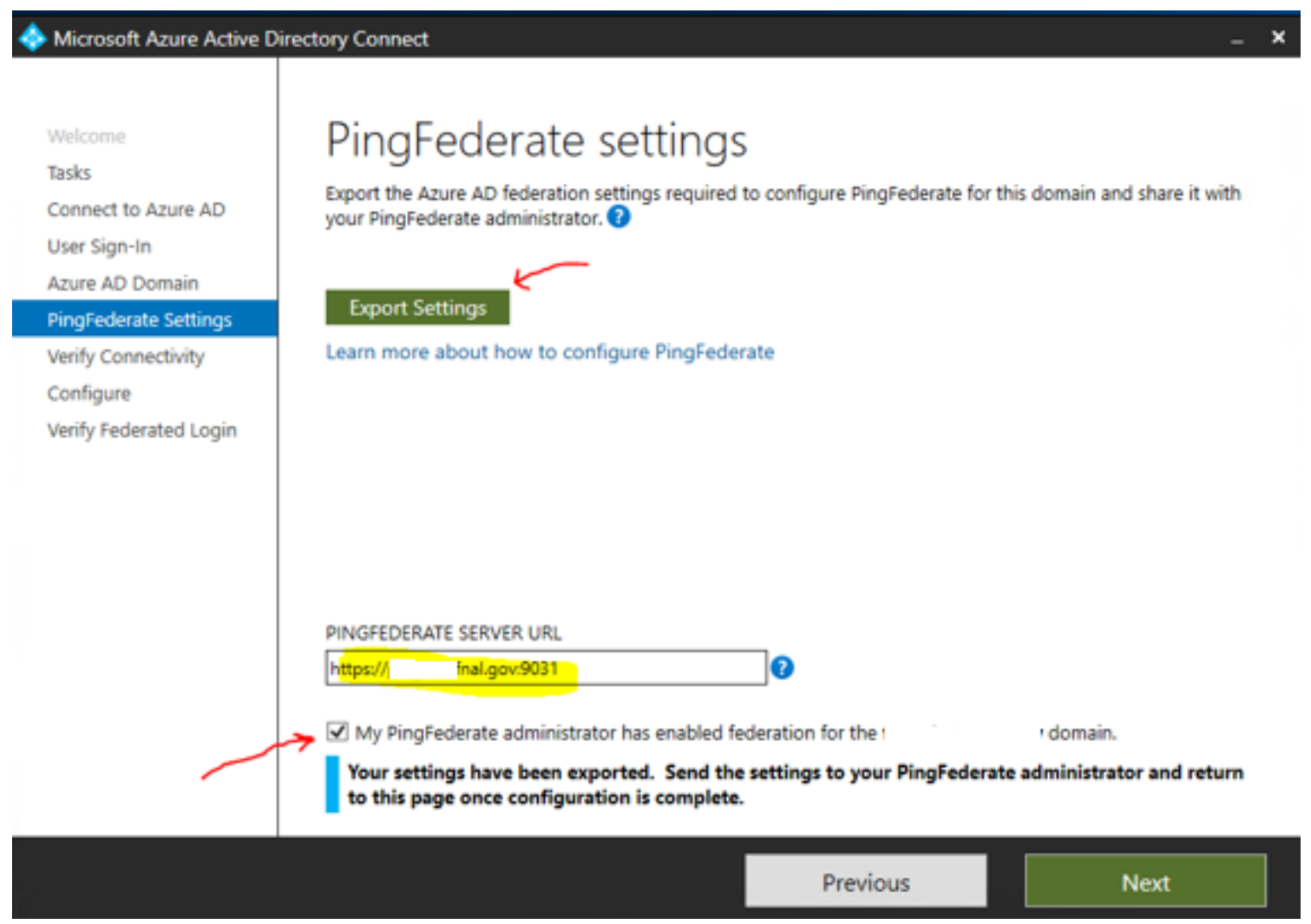




\section{File contents containing Federated Domain Settings for Ping Federate Management Console}

Configuration Parameters from exported Configuration file

Connection types: WS-Federation and WS-Trust

EntityID (Connection ID): "urn:federation:MicrosoftOnline“"

Virtual Server ID: "http://domain.com/PingFederate"

Attribute Contract:

ImmutableID - http://schemas.microsoft.com/LivelD/Federation/2008/05

UPN - http://schemas.xmlsoap.org/claims

Directory attribute source for ImmutableID: "objectGUID" (Binary, Base64)

Directory attribute source for UPN: "userPrincipalName" (String)

Endpoint URL: https://login.microsoftonline.com/login.srf

WS-Trust default token type (PingFederate 8.4 and above): SAML 1.1 for Office 365

WS-Trust token processor type: Username Token Processor 
Populate values from exported File into Ping Federate Management Console

\section{SP Connection}

Connection Type

Connection Options

General Info

Browser SSO

This information identifies your partner's unique connection identifier (Connection ID). Co server IDs for your own server to use when communicating with this partner. If set, these The Base URL may be used to simplify configuration of partner endpoints.

\section{PARTNER'S REALM \\ (CONNECTION ID) \\ CONNECTION NAME}

VIRTUAL SERVER IDS

VIRTUAL SERVER IDS

BASE URL

https://login.microsoftonline.com/login.srf

urn:federation:MicrosoftOnline

Office 365 Test 1

http:

Add
EntityID (Connection ID):

"urn:federation:MicrosoftOnline"

Endpoint URL:

https://login.microsoftonline.com/login.srf
CONTACT EMAIL

APPLICATION NAME

APPLICATION ICON URL

Edit I Delete I

LOGGING MODE

@fnal.gov

Office 365

https://login.microsoftonline.com

NONE

STANDARD

ENHANCED

- FULL
Informational items here

- Contact info

- Application Name

- Application ICON URL

- Logging 


\section{Federate Domain with Ping Federate}

\section{Verify Connectivity}

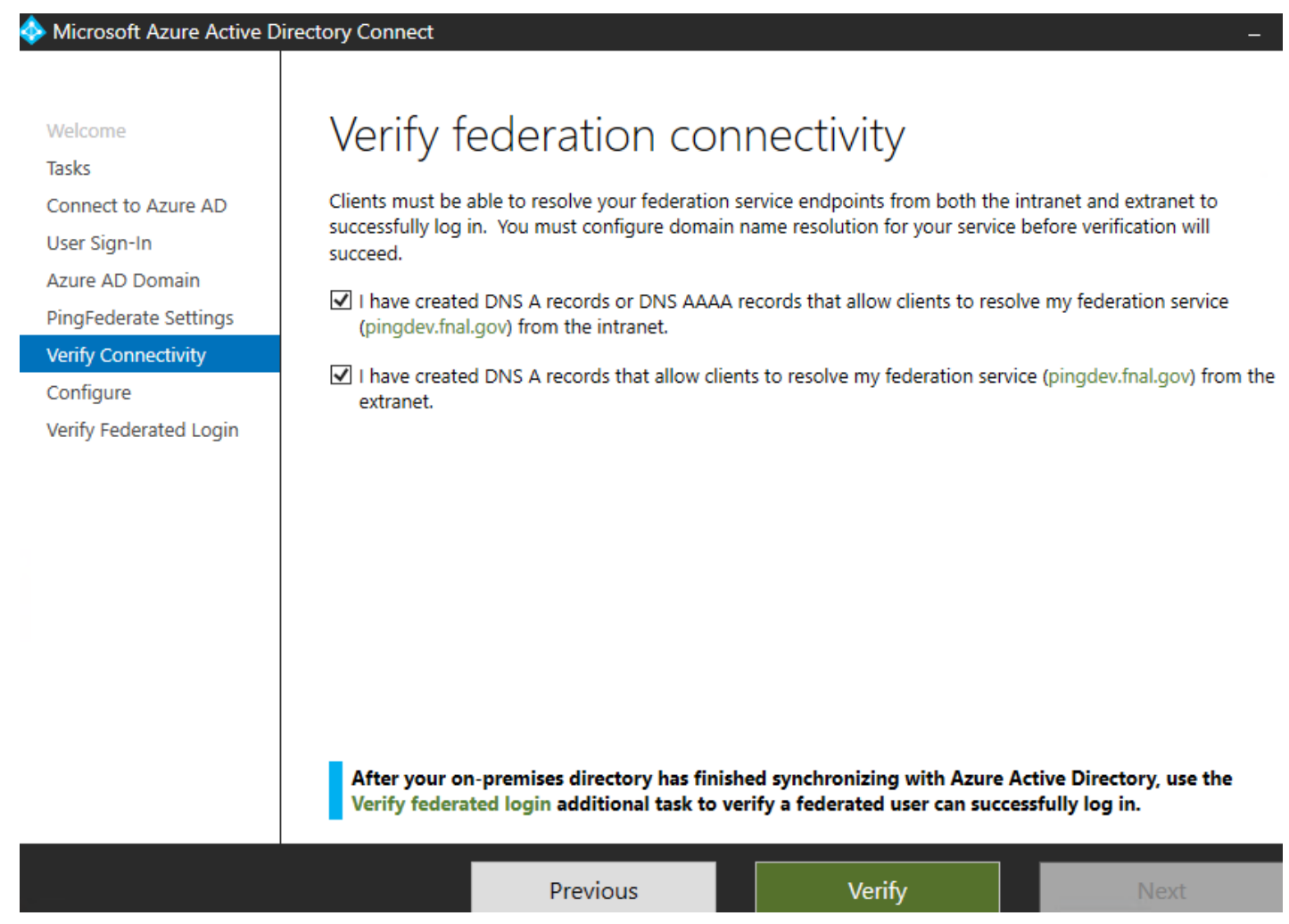

Next $\rightarrow$

Configure Screen just tells what domain you will configure the trust with 


\section{Federate Domain with Ping Federate}

Configuration Complete! You have now Federated with Ping

$\$$ Microsoft Azure Active Directory Connect

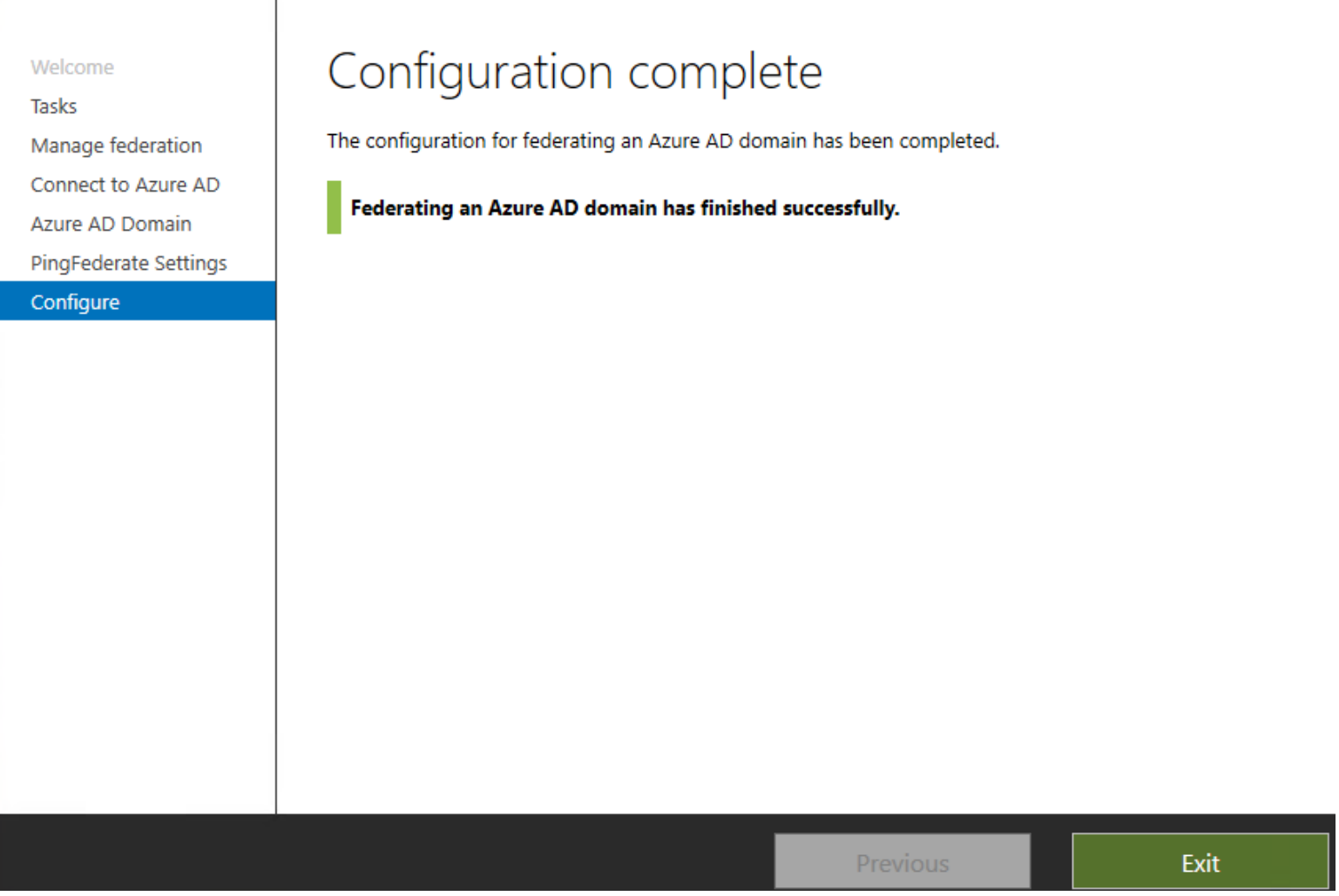

Time to test sign-in! 


\section{Testing}

\section{Operating Systems}

\section{Windows}

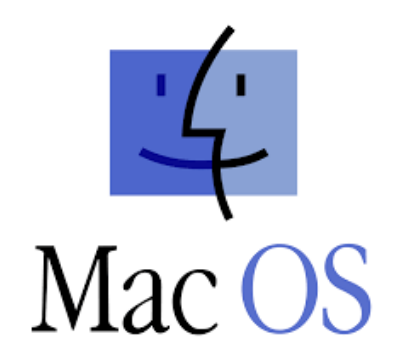

Browsers on MAC

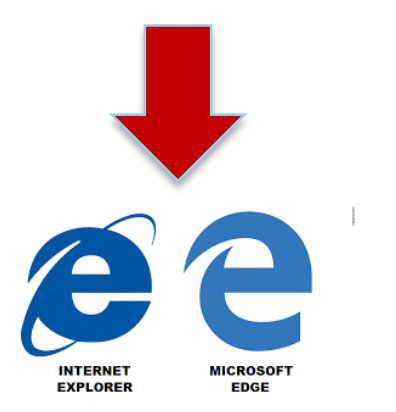

(9) chrome

mozilla

Firefox

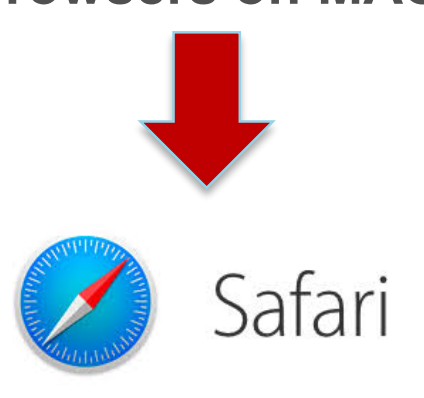

Q chrome

Qchrome

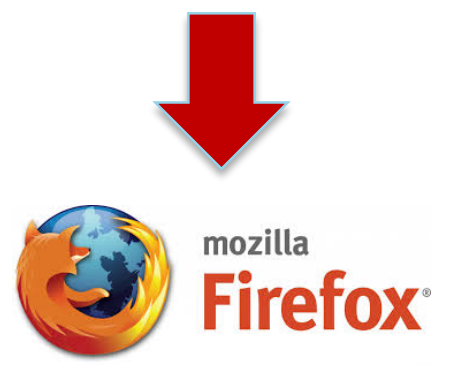

\section{Linux 8}

Browsers on Linux

mozilla

Firefox 


\section{Testing}

\section{Operating Systems}

\section{Windows}

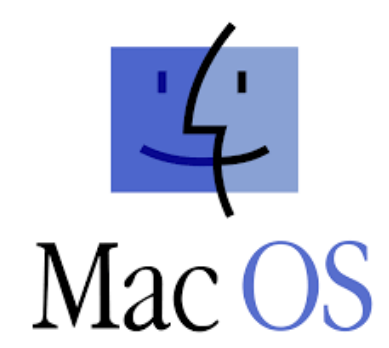

Mail Clients

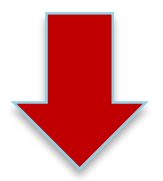

Outlook: $m a c^{2014}$

\section{Linux 8}

Mail Clients
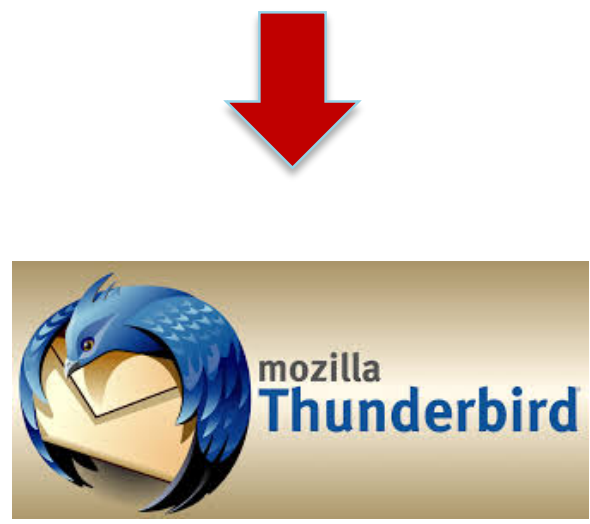

Microsoft Outlook 


\section{Testing}

Android \& IOS Mobile
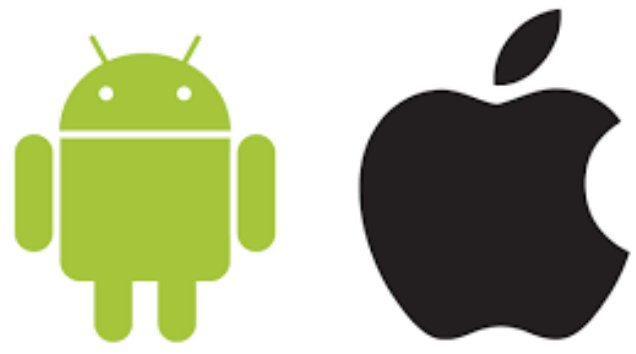

Don't forget Outlook App on both platforms

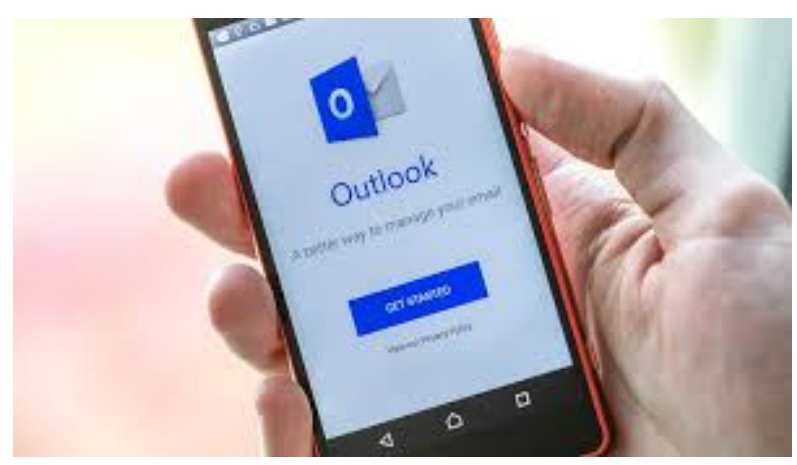

Sign into Office Applications

My account

Kevin J Conway

kconway@

My profile

My account

Sign out

Apps

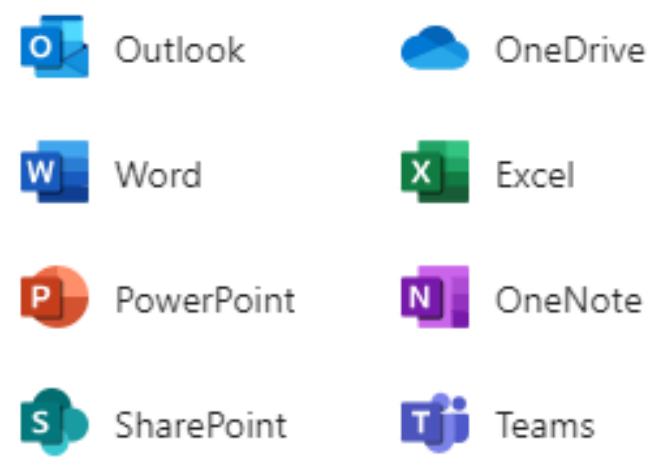




\section{Lessons Learned}

Make sure the WS-TRUST protocol is selected in the Management Console before federating with Ping.

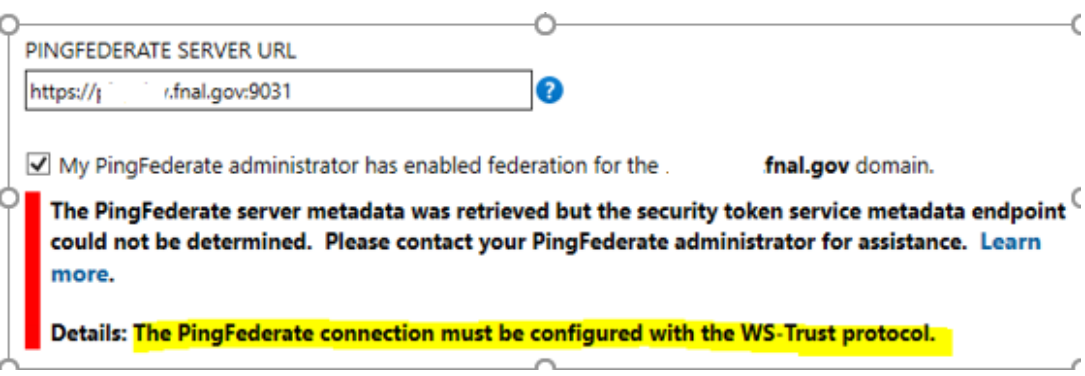

Virtual Server ID value is http://domain/Ping Federate and it is required in the Connection Settings.

PINGFEDERATE SERVER URL
\begin{tabular}{|l|l}
\hline https:// & 9031 \\
\hline
\end{tabular}

$\checkmark$ My Pingfederate administrator has enabled federation for the fnal.gov domain.

The PingFederate server metadata was retrieved but the configuration for .fnal.gov was not

found. Please contact your PingFederate administrator for assistance. Learn more.

Details: Virtual Server ID (http://R

I/PingFederate) not found (500).

You need 2 password Credential Validators for mail clients, mobile phones, \& other active clients

- sAMAccountName $=\$\{$ username $\}$

- userprincipalname $=\$\{$ username $\}$ 


\section{Questions}

